\title{
On equilibrium charge distribution above dielectric surface
}

\author{
D.M.Lytvynenko, Yu.V.Slyusarenko \\ Akhiezer Institute for Theoretical Physics, NSC KIPT, 1 Akademichna Str., 61108 Kharkiv, Ukraine
}

Received December 27, 2008

The problem of the equilibrium state of the charged many-particle system above dielectric surface is formulated. We consider the case of the presence of the external attractive pressing field and the case of its absence. The equilibrium distributions of charges and the electric field, which is generated by these charges in the system in the case of ideally plane dielectric surface, are obtained. The solution of electrostatic equations of the system under consideration in case of small spatial heterogeneities caused by the dielectric surface, is also obtained. These spatial inhomogeneities can be caused both by the inhomogeneities of the surface and by the inhomogeneous charge distribution upon it. In particular, the case of the "wavy" spatially periodic surface is considered taking into account the possible presence of the surface charges.

Key words: charged fermions, surface, solid and liquid dielectrics, equilibrium distribution of charges and electric field

PACS: $05.30 . F k, 05.70 . N p, 41.20 . C v, 71.10 . C a, 73.20 . A t$

\section{Introduction}

The problems concerned with the research of the charges above dielectric surface belong to classical electrodynamics and electrostatics. A special interest to such problems appeared due to the phenomenon of the Wigner crystallization. These studies were initiated in 1934 by Wigner in his theoretical work [1], per se. In this work, the possibility of the existence of periodic structures in the systems with repulsive forces between particles was demonstrated based on the example of crystallization of three-dimensional low-density gas of electrons in the field generated by the spatial-homogeneous positive charge. This field played exactly the role of a compensative factor for repulsive forces. The Landau-Silin Fermi-liquid theory also enables us to predict the existence of spatially-periodic state of electrons in metals and to describe its structure (see in this case [2]). There is still no experimental improvement of Wigners' prediction of the three-dimensional crystallic structures (see, e.g., [3,4]). This is caused by difficulties in achieving the experimental conditions for the mentioned phenomenon, which is also refered to as "Wigner crystallization".

However, as it is well known, different two-dimensional periodic electron structures above the surface of a fluid helium are experimentally realized (the so-called "Wigner crystals"). The works [58] may be referred to as the first publications containing theoretical and real experimental results of different properties of the surface electrons. Numerous works related to the theoretical and experimental research in this area has appeared by now.

Theoretical papers that are devoted to microscopic description of the charge state above dielectric surface are usually based on the conception of an isolated charge above dielectric surface interacting with its electrostatic reflection in dielectric ("levitate electron", see, e.g., [3,4,9]). In this case, the quantum-mechanical state "charge - electrostatic reflection" is described as the hydrogen like one-dimensional state with the corresponding energy structure. Very often the localization of such quantum-mechanical object in the ground state is considered (see $[3-6,9]$ ) occuring at some distance from the surface (first "Bohr radius"). This, particularly in most cases, allows us not to take into account the effect of the surface inhomogeneity on the single charge state. However, in describing the many-particle charge system above dielectric surface the mentioned approach inevitably faces some difficulties. For example, such a difficulty appears when the electron density 
above dielectric surface does not make it possible to consider the charged particles as isolated, i.e., it is necessary to take the interparticle interaction into account.

The references, which are devoted to the two-dimensional Wigner crystallization in the phenomenological approach, predominantly consider the system that consists of a large number of charged particles near the surface of the fluid dielectric as a two-dimensional structure (see, e.g., [3$8])$.

Basing on the premises, it becomes clear that the complete description of charges above dielectric surface needs to take into account their spatial distribution in vacuum. The possibility of adsorption of charges by the surface should also be taken into consideration (in this case the surface inhomogeneities play a crucial role). The possibility of charge spatial distribution above dielectric surface comes from the fact that a charged particle is always attracted by a dielectric surface. Moreover, in the experiments [3,4] concerned with the registration of two-dimensional Wigner crystallization an external electric field attracts charges to the surface and effects their spatial distribution.

The present paper is devoted to the problem of equilibrium charge distribution above dielectric surface both in the external pressing electric field and in its absence. This problem is considered for the case of ideally plane vacuum-dielectric boundary and for the case of "wavy" spatial-periodic surface taking into account the possible existence of the charges "sticked" to the surface. In our opinion, the formulated problem is of great interest both from purely academic and from the research viewpoint concerning the effect of volume charges located closely to the fluid helium surface on the spatial-inhomogeneous state of the charges adsorbed on the helium surface.

\section{Equations of electrostatics for many-charge system above dielectric sur- face}

Let us consider the equilibrium system of charged particles (Fermi-particles) with the charge $Q$ per particle that is situated in vacuum above dielectric surface with the permittivity $\varepsilon$. We describe the profile below the surface by function $\xi(\boldsymbol{\rho}) \equiv \xi(x, y)$, where $\boldsymbol{\rho} \equiv\{x, y\}$ is the radius-vector in the plane $z=0$ of Cartesian coordinates $\{x, y, z\}$. The vacuum - dielectric boundary lies in the plane $z=0$ and we consider it to be unbounded below. All physical quantities considered in the area above the dielectric, i.e., at $z>\xi(\boldsymbol{\rho})$ are marked by the index "1" and all quantities concerned with the dielectric $(z<\xi(\boldsymbol{\rho}))$ are marked by the index " 2 ". Let us assume that the external pressing electric field $E$ acts on the particles and is directed along the z-axis. We also assume the existence of some potential barrier that forbids the charges to penetrate inside the dielectric.

As it is mentioned above, the charged particles are always attracted by the dielectric. Therefore, even in the absence of the external pressing electric field, there is a reason to believe that there are conditions under which the stable equilibrium distribution along the $z$-axis is developed. To avoid the problems of the repulsion of the likely charged particles along the plane $\rho$ we shall consider the system located in a vessel with the walls at $\rho \rightarrow \infty$. These walls prevent the charges from leaving the system. Let us describe the equilibrium charge distribution above the dielectric surface by distribution function $f(\mathbf{p} ; z, \boldsymbol{\rho})$.

The electric field potential $\varphi_{\mathrm{i}}$ in vacuum above the dielectric surface should satisfy the Poisson's equation

$$
\Delta \varphi_{1}(z, \boldsymbol{\rho})=-4 \pi Q n(z, \boldsymbol{\rho}) \theta(z-\xi(\boldsymbol{\rho}))
$$

where $\Delta$ is the Laplace operator,

$$
\Delta \equiv \frac{\partial^{2}}{\partial z^{2}}+\Delta_{\rho}, \quad \Delta_{\rho} \equiv \frac{\partial^{2}}{\partial x^{2}}+\frac{\partial^{2}}{\partial y^{2}},
$$

$\theta(z-\xi(\boldsymbol{\rho}))$ is the Heaviside function. In equation (1) the quantity $n(z, \boldsymbol{\rho})$ is the charge density above the dielectric surface, which can be expressed in terms of the distribution function $f(\mathbf{p} ; z, \boldsymbol{\rho})$ as

$$
n(z, \boldsymbol{\rho})=\int \mathrm{d}^{3} p f(\mathbf{p} ; z, \boldsymbol{\rho}) .
$$


As charges are considered to be Fermi-particles, the distribution function $f(\mathbf{p} ; z, \boldsymbol{\rho})$ has the following form:

$$
f(\mathbf{p} ; z, \boldsymbol{\rho})=\frac{g}{(2 \pi \hbar)^{3}}\left\{\exp \beta\left[\frac{p^{2}}{2 m}+Q \varphi_{1}(z, \boldsymbol{\rho})-\mu\right]+1\right\}^{-1},
$$

where $g=\left(2 S_{Q}+1\right), S_{Q}$ is the spin of the charged particle, $\beta=1 / T, T$ is the temperature in the energy units, $m$ is the charge mass and $\mu$ is the chemical potential of charges. We emphasize that taking into account relations (3) and (4) the equation (1) is often called the Thomas-Fermi equation.

The electric field potential $\varphi_{2}$ is caused by the absence of the charge in the dielectric. In the assumption of the dielectric homogeneity and isotropy it should satisfy the Laplace's equation

$$
\varepsilon \Delta \varphi_{2}(z, \boldsymbol{\rho})=0 .
$$

If the system is placed in the external static homogeneous electric field, the potentials $\varphi_{1}$ and $\varphi_{2}$ can be written in the form

$$
\varphi_{1}=\varphi_{1}^{(i)}+\varphi_{1}^{(e)}, \quad \varphi_{2}=\varphi_{2}^{(i)}+\varphi_{2}^{(e)},
$$

where $\varphi_{1}^{(i)}, \varphi_{2}^{(i)}$ are the potentials induced by the system of charges in vacuum and in the dielectric, respectively, $\varphi_{1}^{(e)}$ and $\varphi_{2}^{(e)}$ are the potentials of the external field in vacuum and in the dielectric. According to equations (1), (5), these fields satisfy the following equations:

$$
\begin{gathered}
\Delta \varphi_{1}^{(i)}(z, \boldsymbol{\rho})=-4 \pi Q n(z, \boldsymbol{\rho}), \quad \Delta \varphi_{1}^{(e)}=0 \\
\Delta \varphi_{2}^{(i)}=0, \quad \Delta \varphi_{2}^{(e)}=0 .
\end{gathered}
$$

Equations (1), (5) for the potentials should be expanded with the boundary conditions on the vacuum-dielectric border (as usual, these conditions can be obtained directly from equations (1), (5), see e.g. [10]):

$$
\begin{gathered}
\left.\varphi_{1}(z, \boldsymbol{\rho})\right|_{z=\xi(\boldsymbol{\rho})}=\left.\varphi_{2}(z, \boldsymbol{\rho})\right|_{z=\xi(\boldsymbol{\rho})}, \\
n_{i}(\boldsymbol{\rho})\left\{\varepsilon \nabla_{i} \varphi_{2}(z, \boldsymbol{\rho})-\varepsilon \nabla_{i} \varphi_{1}(z, \boldsymbol{\rho})\right\}_{z=\xi(\boldsymbol{\rho})}=4 \pi \sigma\left(\boldsymbol{\rho}, \xi\left(\boldsymbol{\rho}^{\prime}\right)\right),
\end{gathered}
$$

where $\mathbf{n}(\boldsymbol{\rho})$ is the unit vector of the surface normal in the point $\boldsymbol{\rho}, \sigma\left(\boldsymbol{\rho}, \xi\left(\boldsymbol{\rho}^{\prime}\right)\right)$ is the surface charge density in the point $\rho$ (here we emphasize the functional dependence of this value on the surface profile $\left.\xi\left(\boldsymbol{\rho}^{\prime}\right)\right)$. The surface charge density $\sigma\left(\boldsymbol{\rho}, \xi\left(\boldsymbol{\rho}^{\prime}\right)\right)$ should satisfy the following relation:

$$
\int \mathrm{d} S_{\xi} \sigma\left(\boldsymbol{\rho}, \xi\left(\boldsymbol{\rho}^{\prime}\right)\right)=Q N_{\xi}
$$

where $N_{\xi}$ is the complete charge number on the dielectric surface and $\mathrm{d} S_{\xi}$ is the surface element with the profile $\xi\left(\boldsymbol{\rho}^{\prime}\right)$ :

$$
\mathrm{d} S_{\xi}=\mathrm{d}^{2} \rho \sqrt{1+(\partial \xi(\boldsymbol{\rho}) / \partial \rho)^{2}} .
$$

The surface charge density can appear for several reasons. For example, these charges may be specially placed on the dielectric surface and can stay there for arbitrarily long time. At that time, the surface charges and the charges above the dielectric surface can differ in sign. But in this case we need to consider the possibility of the formation of bound states of the oppositly charged particles. The presence of such bound states being taken into account represents a separate rather complicated problem. In the present paper this case of surface charges is not considered. The case, when some part of the charges condenses on the surface from the volume distribution, stays for some period of time and then goes back to the volume, is possible too. In this case, the equilibrium distribution of the charges above the surface that coexist with the "sticked" surface charges for some period of time (the lifetime of the charge staying on the surface) is possible. Next, we shall take into account only the possible presence of the surface charges that have the same sign as the volume ones. 
It is easy to see that in equation (8) the directional cosines of the surface normal vector $\mathbf{n}(\boldsymbol{\rho})$ in the point $\rho$ play the main role: $\cos \nu$ is cosine of the angle between the normal and z-axis, $\cos \lambda$ is cosine of the angle between the normal and $\mathrm{x}$-axis and $\cos \mu$ is cosine of the angle between the normal and y-axis. In the case when the surface profile is given explicitly (in our case $z=\xi(\boldsymbol{\rho})$ ), these cosines are determined by the following relations:

$$
\begin{aligned}
\cos \nu & =\frac{1}{\sqrt{1+(\partial \xi(\boldsymbol{\rho}) / \partial \rho)^{2}}}, \quad \cos \lambda=-\frac{\partial \xi(\boldsymbol{\rho}) / \partial x}{\sqrt{1+(\partial \xi(\boldsymbol{\rho}) / \partial \rho)^{2}}}, \\
\cos \mu & =-\frac{\partial \xi(\boldsymbol{\rho}) / \partial y}{\sqrt{1+(\partial \xi(\boldsymbol{\rho}) / \partial \rho)^{2}}} .
\end{aligned}
$$

The derived electrostatics equations (1), (5) with the boundary conditions (8), (11) can be analytically solved in a very low case count. Some of these cases are considered below. Before solving equations (1), (5), let us consider the simplification of the boundary conditions (8) in the case, when the surface profile differs a little from the plane one. In this case we essentially have the effective boundary conditions. From equations (10), (11) it is obvious that the surface slightly differs from the plane one, when the surface profile slowly varies on the coordinate, i.e., when the following inequalities take place:

$$
|\partial \xi(\boldsymbol{\rho}) / \partial x| \ll 1, \quad|\partial \xi(\boldsymbol{\rho}) / \partial y| \ll 1 .
$$

Let us also consider that the surface profile $\xi(\boldsymbol{\rho})$ can be presented as:

$$
\xi(\boldsymbol{\rho})=\xi+\tilde{\xi}(\boldsymbol{\rho}), \quad|\xi| \gg|\tilde{\xi}(\boldsymbol{\rho})|
$$

It is easy to see that the inequality (12) is provided in this case by the conditions

$$
|\partial \tilde{\xi}(\boldsymbol{\rho}) / \partial x| \ll 1, \quad|\partial \tilde{\xi}(\boldsymbol{\rho}) / \partial y| \ll 1
$$

The directional cosines (11), with the accuracy up to the second order over $\partial \widetilde{\xi}(\boldsymbol{\rho}) / \partial \boldsymbol{\rho}$, have the following form:

$$
\cos \nu \approx 1, \quad \cos \lambda=-\partial \widetilde{\xi}(\boldsymbol{\rho}) / \partial x, \quad \cos \mu=-\partial \widetilde{\xi}(\boldsymbol{\rho}) / \partial y
$$

If the relations (13)-(15) take place, we can expect that the charge and the field distributions in the system slightly differ from the distributions that take place in the case of the plane dielectric surface. Then, the potentials $\varphi_{1}(z, \boldsymbol{\rho})$ and $\varphi_{2}(z, \boldsymbol{\rho})$ (see equations $\left.(1),(5)\right)$ can be written as

$$
\varphi_{1}(z, \boldsymbol{\rho})=\varphi_{1}(z)+\widetilde{\varphi}_{1}(z, \boldsymbol{\rho}), \quad \varphi_{2}(z, \boldsymbol{\rho})=\varphi_{2}(z)+\widetilde{\varphi}_{2}(z, \boldsymbol{\rho}),
$$

where $\varphi_{1}(z)$ and $\varphi_{2}(z)$ are the potentials of some electric field above the dielectric and inside it (but not on the surface!) in the case of the plane surface. The small distortions of the field above the dielectric and inside it are described by the potentials $\widetilde{\varphi}_{1}(z, \boldsymbol{\rho})$ and $\widetilde{\varphi}_{2}(z, \boldsymbol{\rho})$ due to the surface inhomogeneity in the above mentioned sense. The meaning of the introduced potentials $\varphi_{1}(z)$ and $\varphi_{2}(z)$, as well as $\widetilde{\varphi}_{1}(z, \boldsymbol{\rho})$ and $\widetilde{\varphi}_{2}(z, \boldsymbol{\rho})$ becomes more clear after obtaining the Poisson's equations and effective boundary conditions for them.

According to the assumption of small field pertrubations provided by the wave surface, the following inequalities take place:

$$
\left|\varphi_{1}(z)\right| \gg\left|\tilde{\varphi}_{1}(z, \boldsymbol{\rho})\right|, \quad\left|\varphi_{2}(z)\right| \gg\left|\tilde{\varphi}_{2}(z, \boldsymbol{\rho})\right| .
$$

Let us also consider that the distribution of charges that can be condensed on the surface slightly differs from the homogeneous one:

$$
\begin{aligned}
\sigma(\boldsymbol{\rho}, \xi) & =\sigma(\xi)+\widetilde{\sigma}(\boldsymbol{\rho}, \xi)+\frac{\partial \sigma(\xi)}{\partial \xi} \widetilde{\xi}(\boldsymbol{\rho}) \\
|\sigma(\xi)| & \gg|\tilde{\sigma}(\boldsymbol{\rho} ; \xi)|,|\sigma(\xi)| \gg\left|\frac{\partial \sigma(\xi)}{\partial \xi} \tilde{\xi}(\boldsymbol{\rho})\right| .
\end{aligned}
$$


In the expressions (18) the quantity $\widetilde{\sigma}(\boldsymbol{\rho}, \xi)$ corresponds to the impact of the weakly inhomogeneous charge distribution on the plane dielectric surface with $z=\xi$ profile. The quantity $\widetilde{\xi}(\boldsymbol{\rho})[\partial \sigma(\xi) / \partial \xi]$ in equation (18) describes the surface charge inhomogeneity related to the weak irregularity of the surface itself.

In the expressions (16), (17) we consider that in the case of uniformly charged surface, which is ideally plane and infinitely extended with charge density $\sigma(\xi)$ both the field and the charge distributions are homogeneous along $\rho$ plane. In other words, the spatial charge and field distribution depend only on z coordinates.

From the relations (13)-(18) it is easy to obtain the effective boundary conditions for field potentials on vacuum-dielectric boundary in the case, when the dielectric surface weakly differs from the ideally plane one. To this end, we should develop the perturbation theory over small values $\widetilde{\xi}(\boldsymbol{\rho}), \widetilde{\sigma}(\boldsymbol{\rho}, \xi)$ and $\partial \widetilde{\xi}(\boldsymbol{\rho}) / \partial \boldsymbol{\rho}$, which, according to the expressions (12)-(18), can be given as follows:

$$
\begin{aligned}
& \left\{\varphi_{1}(z)+\widetilde{\varphi}_{1}(z, \boldsymbol{\rho})\right\}_{\xi+\widetilde{\xi}(\boldsymbol{\rho})}=\left\{\varphi_{2}(z)+\widetilde{\varphi}_{2}(z, \boldsymbol{\rho})\right\}_{\xi+\widetilde{\xi}(\boldsymbol{\rho})}, \\
& \left\{\varepsilon \frac{\partial}{\partial z}\left[\varphi_{2}(z)+\widetilde{\varphi}_{2}(z, \boldsymbol{\rho})\right]-\frac{\partial}{\partial z}\left[\varphi_{1}(z)+\widetilde{\varphi}_{1}(z, \boldsymbol{\rho})\right]\right\}_{\xi+\widetilde{\xi}(\boldsymbol{\rho})}=4 \pi \sigma(\boldsymbol{\rho} ; \xi+\widetilde{\xi}(\boldsymbol{\rho})) .
\end{aligned}
$$

Making the necessary calculations up to the first order of the perturbation theory from the first relation of equation (19) we obtain

$$
\left.\varphi_{1}(z)\right|_{z=\xi}=\left.\varphi_{2}(z)\right|_{z=\xi}, \quad\left\{\widetilde{\varphi}_{1}(z, \boldsymbol{\rho})-\widetilde{\varphi}_{2}(z, \boldsymbol{\rho}),\right\}_{z=\xi}=\left\{\frac{\partial \varphi_{2}(z)}{\partial z}-\frac{\partial \varphi_{1}(z)}{\partial z}\right\}_{z=\xi} \widetilde{\xi}(\boldsymbol{\rho}) .
$$

The use of the perturbation theory up to the first order for the second relation of equation (19) results in the following equalities:

$$
\begin{aligned}
& \left\{\varepsilon \frac{\partial \varphi_{2}(z)}{\partial z}-\frac{\partial \varphi_{1}(z)}{\partial z}\right\}_{z=\xi}=4 \pi \sigma(\xi) \\
& \left\{\varepsilon \frac{\partial^{2} \varphi_{2}(z)}{\partial z^{2}}-\frac{\partial^{2} \varphi_{1}(z)}{\partial z^{2}}-4 \pi \frac{\partial \sigma(\xi)}{\partial \xi}\right\}_{z=\xi} \widetilde{\xi}(\boldsymbol{\rho})-4 \pi \widetilde{\sigma}(\boldsymbol{\rho} ; \xi)=\left\{\frac{\partial \widetilde{\varphi}_{1}(z, \boldsymbol{\rho})}{\partial z}-\varepsilon \frac{\partial \widetilde{\varphi}_{2}(z, \boldsymbol{\rho})}{\partial z}\right\}_{z=\xi}
\end{aligned}
$$

Let us recall that in electrostatics the denotations like $\left\{\partial^{2} \varphi_{1}(z) / \partial z^{2}\right\}_{z=\xi},\left\{\partial^{2} \varphi_{2}(z) / \partial z^{2}\right\}_{z=\xi}$ have the meaning of limits

$$
\begin{aligned}
& \left\{\partial^{2} \varphi_{1}(z) / \partial z^{2}\right\}_{z=\xi}=\lim _{h \rightarrow 0}\left\{\partial^{2} \varphi_{1}(z) / \partial z^{2}\right\}_{z=\xi+h}, \\
& \left\{\partial^{2} \varphi_{2}(z) / \partial z^{2}\right\}_{z=\xi}=\lim _{h \rightarrow 0}\left\{\partial^{2} \varphi_{2}(z) / \partial z^{2}\right\}_{z=\xi-h} .
\end{aligned}
$$

Then, for a further simplification of the obtained effective boundary conditions (20), (21), according to equations $(1),(5),(16)$ we can use the following equations, which are satisfied by the potentials $\varphi_{1}(z), \varphi_{2}(z)$ :

where

$$
\frac{\partial^{2}}{\partial^{2}} \varphi_{1}(z)=-4 \pi Q n(z) \theta(z-\xi), \quad \frac{\partial^{2}}{\partial^{2}} \varphi_{2}(z)=0
$$

$$
n(z)=\int \mathrm{d}^{3} p f(\mathbf{p}, z), \quad f(\mathbf{p} ; z)=\frac{g}{(2 \pi \hbar)^{3}}\left\{\exp \beta\left[\frac{p^{2}}{2 m}+Q \varphi_{1}(z)-\mu\right]+1\right\}^{-1} .
$$

Taking into account equation (21), the conditions (20) can be expressed in the following form (the first one remains the same):

$$
\begin{aligned}
& \left\{\varepsilon \frac{\partial \varphi_{2}(z)}{\partial z}-\frac{\partial \varphi_{1}(z)}{\partial z}\right\}_{z=\xi}=4 \pi \sigma(\xi) \\
& 4 \pi\left\{Q n(z)-\frac{\partial \sigma(\xi)}{\partial \xi}\right\}_{z=\xi} \widetilde{\xi}(\boldsymbol{\rho})-4 \pi \widetilde{\sigma}(\boldsymbol{\rho} ; \xi)=\left\{\frac{\partial \widetilde{\varphi}_{1}(z, \boldsymbol{\rho})}{\partial z}-\varepsilon \frac{\partial \widetilde{\varphi}_{2}(z, \boldsymbol{\rho})}{\partial z}\right\}_{z=\xi} .
\end{aligned}
$$


Thus, we obtain effective boundary conditions (20), (24) for the fields in the system of charges above the dielectric surface with the surface profile that slightly differs from the plane one:

$$
\begin{aligned}
& \frac{\partial^{2} \widetilde{\varphi}_{1}(z, \boldsymbol{\rho})}{\partial z^{2}}+\Delta_{\boldsymbol{\rho}} \widetilde{\varphi}_{1}(z, \boldsymbol{\rho})=4 \pi Q^{2} \frac{\partial n(z)}{\partial \mu} \widetilde{\varphi}_{1}(z, \boldsymbol{\rho}), \\
& \frac{\partial^{2} \widetilde{\varphi}_{2}(z, \boldsymbol{\rho})}{\partial z^{2}}+\Delta_{\boldsymbol{\rho}} \widetilde{\varphi}_{2}(z, \boldsymbol{\rho})=0 .
\end{aligned}
$$

\section{System of charges above the ideally plane dielectric surface}

It is easy to see that the obtained equations (22), (23) of electrostatics and the effective boundary conditions (20), (24) for these equations are much simpler than the initial electrostatic equations (1), (5) and than the boundary conditions (8). Firstly, to solve the equations that determine the charge and the field distribution above the vacuum-dielectric boundary one needs to consider the case of an ideally plane surface of this boundary that lies in $z=\xi$. Let us start withe considering the case of the surface in the absence of the charge $\sigma=0$. Then, the solution of (22) should satisfy the following boundary conditions:

$$
\left.\varphi_{1}(z)\right|_{z=\xi}=\left.\varphi_{2}(z)\right|_{z=\xi}, \quad\left\{\varepsilon \frac{\partial \varphi_{2}(z)}{\partial z}-\frac{\partial \varphi_{2}(z)}{\partial z}\right\}_{z=\xi}=0 .
$$

To simplify the further calculations, let us write the first formula from equation (22) in the following form:

$$
\frac{\partial \varphi_{1}^{2}(z)}{\partial z^{2}}=-4 \pi Q \nu \int_{0}^{\infty} \mathrm{d} \varepsilon \varepsilon^{1 / 2}\{\exp \beta(\varepsilon-\psi)+1\}
$$

where we denote

$$
\psi(z) \equiv \mu-Q \varphi_{1}(z), \quad \nu \equiv(2 m)^{3 / 2} / 2 \pi^{2} \hbar^{3} .
$$

Here we also consider the spin of a charged particle equal to $1 / 2, \psi$ is the so-called electrochemical potential.

Multiplying equation (27) by the derivative $\left(\partial \varphi_{1}(z) / \partial z\right)$ and using the following equality

$$
\left(\frac{\partial \varphi_{1}}{\partial z}\right) \frac{1}{\mathrm{e}^{\beta(\varepsilon-\psi)}+1}=-\frac{1}{\beta Q} \frac{\partial}{\partial z} \ln \left[\mathrm{e}^{-\beta(\varepsilon-\psi)}+1\right]
$$

after simple calculations we obtain the first-order differential equation:

$$
\left(\frac{\partial \varphi_{1}}{\partial z}\right)^{2}=\frac{16 \pi}{3} \nu \int_{0}^{\infty} \mathrm{d} \varepsilon \varepsilon^{3 / 2}\left\{\mathrm{e}^{\beta(\varepsilon-\psi)}+1\right\}^{-1}+C,
$$

where $C$ is an arbitrary integration constant. Thus, there arises the need to solve the equation as follows:

$$
\frac{\partial \varphi_{1}}{\partial z}= \pm\left\{\frac{16 \pi}{3} \nu \int_{0}^{\infty} \mathrm{d} \varepsilon \varepsilon^{3 / 2}\left\{\mathrm{e}^{\beta(\varepsilon-\psi)}+1\right\}^{-1}+C\right\}^{1 / 2} .
$$

The sign before the square root in equation (29) should be chosen on the following considerations. The force acting on the charges at $z>\xi$ presses these charges to a dielectric surface. Thus, in the case of positive charges above the dielectric we choose the positive sign, and in the case of negative charges we choose the negative sign. Let us consider the distribution of negative charges above the dielectric surface, $Q=-\mathrm{e}$, e $>0$. Hence, the potential $\varphi_{1}$ satisfies the relation:

$$
\frac{\partial \varphi_{1}}{\partial z}=-\left\{\frac{16 \pi}{3} \nu \int_{0}^{\infty} \mathrm{d} \varepsilon \varepsilon^{3 / 2}\left\{\mathrm{e}^{\beta(\varepsilon-\psi)}+1\right\}^{-1}+C\right\}^{1 / 2} .
$$


Now we make the following denotations:

$$
\varphi_{1}(z=0) \equiv \varphi_{0}, \quad \psi(z=0) \equiv \mu+e \varphi_{1}(z=0), \quad E_{0} \equiv-\left(\frac{\partial \varphi_{1}(z)}{\partial z}\right)_{z=0} .
$$

Let us recall that we consider the case of the electric forces that attract charges to the dielectric surface. Thus, at $z \rightarrow \infty$ there are no charges, $f(\mathbf{p} ; z) \underset{z \rightarrow \infty}{\longrightarrow} 0$, or

$$
\{\exp \beta(\varepsilon-\psi)+1\}^{-1} \underset{z \rightarrow \infty}{\rightarrow} 0 .
$$

The action of the electrostatic image force along z-axis must vanish at $z \rightarrow \infty$ :

$$
\frac{\partial \varphi_{1}^{(i)}(z)}{\partial z} \underset{z \rightarrow \infty}{\rightarrow} 0
$$

As the result, it is essential to say that at $z \rightarrow \infty$ the following relation takes place:

$$
-\frac{\partial \varphi_{1}(z)}{\partial z} \underset{z \rightarrow \infty}{\rightarrow}-\frac{\partial \varphi_{1}^{(e)}(z)}{\partial z} \equiv E
$$

where $E$ is the external field intensity that attracts the charges to the dielectric surface.

At $z=0$ from equation (30) one can get

$$
E_{0}^{2}=\frac{16 \pi}{3} \nu \int_{0}^{\infty} \mathrm{d} \varepsilon \varepsilon^{3 / 2}\left\{\exp \beta\left(\varepsilon-\psi_{0}\right)+1\right\}^{-1}+C
$$

On the other hand, from the same equation and taking into account equations (32), (33) at $z \rightarrow \infty$ we obtain:

$$
C=E^{2}
$$

Comparing the last two expressions, we come to the relation between the constants $\psi_{0}, E_{0}$ (see equation (31)) and the external electromagnetic field $E$ :

$$
E_{0}^{2}-E^{2}=\frac{16 \pi}{3} \nu \int_{0}^{\infty} \mathrm{d} \varepsilon \varepsilon^{3 / 2}\left\{\exp \beta\left(\varepsilon-\psi_{0}\right)+1\right\}^{-1}
$$

Then, after integrating the first expression from equation (23) over $\mathrm{z}$ within the limits from $\xi$ to $\varepsilon$ and using equations (32), (34), we get:

$$
E_{0}-E=4 \pi \mathrm{e} n_{s}, \quad \mathrm{e}>0,
$$

where $n_{s}$ is the number of the volume charges per unit of the plane dielectric surface:

$$
n_{s}=\int_{\xi}^{\infty} \mathrm{d} z n(z), \quad n(z)=\nu \int_{0}^{\infty} \mathrm{d} \varepsilon \varepsilon^{1 / 2}\{\exp \beta(\varepsilon-\psi(z))+1\}^{-1} .
$$

Let us emphasize that for the equilibrium charge system above dielectric the value of the number $n_{s}$ depends neither on the coordinates nor on the fields' distribution. This is determined only by the entire number $N$ of the charges above dielectric. We also point out that this value characterizes the additional field intensity that presses the charges to the dielectric surface. Besides that, this field is generated by the charges themselves.

Thus, equations (35), (36) make it possible to express the unknown quantities $\psi_{0}$ and $E_{0}$ (integration constants of equation (27)) in terms of the external pressing electric field $E$ and the number of charges above the unit item of the dielectric surface $n_{s}$ (see equation (37)). 
The second equation in (38) can be solved trivially in general case, because the electric field intensity in the dielectric does not depend on $z$. Using the boundary conditions (26), we can express the potential of the electric field in the dielectric in the following form:

$$
\varphi_{2}=-\frac{E_{0}}{\varepsilon} z+\varphi_{0}, \quad E_{2}=\frac{E_{0}}{\varepsilon},
$$

where $E_{2}$ is the electric field intensity in the dielectric, $E_{0}$ can be expressed from equations (35), (36) and $\varphi_{0}$ is the potential on the surface. Like in the electrostatic case, the field potential is determined accurate within a constant. Therefore, we set the potential $\varphi_{0}$ equal to zero below. In this case, the value of the electrochemical potential on the dielectric surface coincides with the chemical one:

$$
\psi(z=0) \equiv \psi_{0}=\mu, \quad \varphi_{0}=0 .
$$

Taking into account equation (34), the spatial distribution of the potential (see equation (30)) can be written as follows:

$$
\frac{\partial \varphi_{1}}{\partial z}=-\left\{\frac{16 \pi}{3} \nu \int_{0}^{\infty} \mathrm{d} \varepsilon \varepsilon^{3 / 2}\{\exp \beta(\varepsilon-\psi)+1\}^{-1}+E^{2}\right\}^{1 / 2}, \quad \psi(z) \equiv \mu+e \varphi_{1}(z) .
$$

It is easy to see that in a general case the solution of this equation can be found only in quadratures (see below). However, the gas of charged Fermi-particles above the dielectric surface is nondegenerate. Therefore, the solution of equation (40) can be obtained analytically. Indeed, in the case of nondegenerate gas its distribution function has the form that weakly differs from the Boltzmans' one

$$
\{\exp \beta(\varepsilon-\psi)+1\}^{-1} \sim \exp \beta(\psi-\varepsilon) .
$$

Accordingly, the expression for the density distribution of a gas along the $z$ coordinate (see equation (37)) becomes:

$$
n(z) \approx \frac{\sqrt{\pi}}{2} \nu \beta^{-3 / 2} \exp (\beta \psi)
$$

As the Fermi-particle gas is degenerate at low temperature and at high density ranges (see e.g. [11]) from equation (41) one can get the gas nondegeneracy condition:

$$
\exp (\beta \psi) \ll 1
$$

As the electrochemical potential depends on $\mathrm{z}$, this condition is obviously realized in the case when the following inequality takes place:

$$
\exp \left(\beta \psi_{0}\right) \ll 1
$$

where $\psi_{0}$ is the electrochemical potential on the dielectric surface (see equations (28), (39) in this case). The latter statement takes place due to the assumption of the particle absence at $z \rightarrow \infty$, see above. So, according to equation (41), (42), the formula (40) can be expressed as follows:

$$
\frac{\partial \psi}{\partial z}=-\left\{4 \pi^{3 / 2} \mathrm{e}^{2} \beta^{-5 / 2} \nu \exp (\beta \psi)+\mathrm{e}^{2} E^{2}\right\}^{1 / 2}
$$

This equation has an analytical solution:

$$
\frac{\sqrt{\pi}}{2} \nu \beta^{-3 / 2} \exp (\beta \psi(z))=\beta \frac{E^{2}}{8 \pi} \frac{4 \chi(z)}{(1-\chi(z))^{2}}, \quad \psi(z) \equiv \mu+e \varphi_{1}(z)
$$

where the function $\chi(z)$ is defined by the relation:

$$
\chi(z) \equiv \frac{E_{0}-E}{E_{0}+E} \exp \left\{-(z-\xi) / z_{0}\right\}, \quad z_{0} \equiv(\beta e E)^{-1}, \quad \beta^{-1}=T .
$$


Let us emphasize that the multiplier before the exponent in equation (45) according to equation (36) can be expressed in terms of the intensity of the external electric field and the number of charges $n_{s}$ in the "column" above the surface unit element:

$$
\frac{E_{0}-E}{E_{0}+E}=\frac{2 \pi \mathrm{e} n_{s}}{E+2 \pi \mathrm{e} n_{s}}
$$

From the equations (41), (43) and (44) it follows that the charge density above the dielectric surface has the distribution:

$$
n(z)=\beta \frac{E^{2}}{8 \pi} \frac{4 \chi(z)}{(1-\chi(z))^{2}},
$$

and the electric field intensity above the dielectric $E_{1}(z)$ is expressed as

$$
E_{1}(z)=E \frac{1+\chi(z)}{1-\chi(z)}
$$

It is easy to see that at high values of $z, z \gg z_{0}$ (see equation (45)), the charge distribution above the dielectric surface is close to the Boltzman distribution and the electric field density exponentially tends to the external pressing electric field density. This fact confirms the above assumptions(see equations (32), (33)).

The inequality (42) that determines the nondegeneracy condition of the charge gas can be written in terms of the obtained solutions:

$$
\mathrm{e} n_{s} \nu^{-1} \beta^{5 / 2}\left(E+2 \pi \mathrm{e} n_{s}\right) \ll 1 .
$$

It is obvious that this inequality is not accomplished in the case of low temperature range or high values of the external pressing field.

Expressions (44)-(48) make it possible to make the limit process at $E \rightarrow 0$. In the case of the absence of the external pressing field, these solutions have the following form:

$$
E_{1}(z) \underset{E \rightarrow 0}{\rightarrow} E_{0}\left\{1+\frac{z-\xi}{2 z_{0}}\right\}^{-1}, \quad n(z) \underset{E \rightarrow 0}{\rightarrow} \beta \frac{E_{0}^{2}}{8 \pi}\left\{1+\frac{z-\xi}{2 z_{0}}\right\}^{-2}, \quad E_{2}=E_{0} / \varepsilon
$$

where

$$
z_{0} \equiv\left(\beta e E_{0}\right)^{-1}, \quad E_{0}=4 \pi \mathrm{e} n_{s} .
$$

Comparing the expressions (46), (47) and (49), it is easy to see that in the case of the absence of the external pressing field, the exponential law of the electric field and the charge density above the dielectric surface changes to the weaker power depedence. In this case, the inequality (48) can be written as:

$$
\left(\mathrm{e} n_{s}\right)^{2} \nu^{-1} \beta^{5 / 2} \ll 1 .
$$

Note, that this takes place in the region of relatively high temperatures and low charge number in the volume above a surface area unit, see equation (37).

The obtained formulae (38), (44)-(51) are the solution of the problem of the field and nondegenerate charged gas distribution in charged particle system above the plane dielectric surface both in the external pressing field and in its absence. Let us emphasize that the dielectric permittivity does not appear in these expressions. The reason is that the problem is homogeneous along the surface coordinate $\rho$. In the case of inhomogeneity along $\rho$, the solution of equations essentially depends on the sort of the dielectric, i.e., on its permittivity $\varepsilon$. These inhomogeneities may be caused by the inhomogeneities of the surface itself or by inhomogeneity charge distribution on it (or both reasons simultaneously, see equations (8), (11), (20), (24)).

In the case of the degenerate gas, i.e., when the condition (48) or (51) fails, the solution that is obtained earlier is inapplicable. Let us make the following remark relative to this fact. As it is mentioned earlier, the charge density distribution decreases with the distance from the surface. For this reason, in a general case described by equation (40) the gas can be degenerate in the area 
near the dielectric surface and nondegenerate far from it. The typical distance from the surface that separates these cases can be obtained using the following considerations. As it is well known (see e. g. [11]), in low temperature region, the temperature expansions are widely used for the calculus of the thermodynamic quantities characterizing the gas. Applying such an expansion to the integral over the energy in equation (40), we obtain:

$$
\int_{0}^{\infty} \mathrm{d} \varepsilon \varepsilon^{3 / 2}\{\exp \beta(\varepsilon-\psi)+1\}^{-1} \approx \frac{2}{5} \psi^{5 / 2}+\frac{\pi^{2}}{4} \beta^{-2} \psi^{1 / 2}-\frac{7 \pi^{4}}{960} \beta^{-4} \psi^{-3 / 2}+\cdots
$$

From this expression it is easy to see that such an expansion is absolutely useless near the point $z_{1}$ obtained from the condition

$$
\psi\left(z_{1}\right)=\mu+e \varphi_{1}\left(z_{1}\right)=0 .
$$

The solution of equation (40) obtained in quadratures is given by

$$
z-\xi=-z_{0} \int_{\beta \psi_{0}}^{\beta \psi} \mathrm{d} \zeta\left\{\frac{16 \pi}{3} \nu \beta^{-5 / 2} E^{-2} \int_{0}^{\infty} \mathrm{d} y y^{3 / 2}\{\exp (y-\zeta)+1\}^{-1}+1\right\}^{-1 / 2}
$$

where the distance $z_{0}$ is determined by equation (45). Taking into account equations (53), (54), the expression of the border distance $z_{1}$ can be written as follows:

$$
z_{1}=\xi+z_{0} \int_{0}^{\beta \psi_{0}} \mathrm{~d} \zeta\left\{\frac{16 \pi}{3} \nu \beta^{-5 / 2} E^{-2} \int_{0}^{\infty} \mathrm{d} y y^{3 / 2}\{\exp (y-\zeta)+1\}^{-1}+1\right\}^{-1 / 2}
$$

where the electrochemical potential $\psi_{0}$ as the function of temperature $T=\beta^{-1}$ and the external electric field is obtained from the equation (see equations (35), (36), (39))

$$
\left(4 \pi \mathrm{e} n_{s}+E\right)^{2}-E^{2}=\frac{16 \pi}{3} \nu \int_{0}^{\infty} \mathrm{d} \varepsilon \varepsilon^{3 / 2}\left\{\exp \beta\left(\varepsilon-\psi_{0}\right)+1\right\}^{-1} .
$$

As it is mentioned above, the potential $\varphi_{1}(z)$ is defined accurate within an arbitrary constant, which can be set equal to zero. Hence, equation (56) is the expression defining the chemical potential $\mu$, see equation (39).

As expected, the typical distance $z_{1}$ (see equation (55)) is defined by the temperature, the external pressing field and the number of charges above the dielectric surface area unit. Thus, the charge gas is nondegenerate in the region $z \gg z_{1}$ and degenerate at $z \ll z_{1}$. Let us point out that the solutions (45)-(50) are obtained assuming the charge gas nondegeneracy in the entire area above the dielectric surface. Therefore, in a general case the mentioned expressions describe the charge system only in the region $z \gg z_{1}$. The charge gas above the dielectric surface can be degenerate even in the case of the absence of the external pressing field. It is easy to see whether it is necessary to analyze the expression (55) at $E \rightarrow 0$ taking equation (45) into account.

In the case of the generate charge gas above dielectric surface, equation (40) according to equation (52) can be written in a simpler form:

$$
\frac{\partial \varphi_{1}}{\partial z}=-\left\{\frac{32 \pi}{15} \nu \psi^{5 / 2}+E^{2}\right\}^{1 / 2}, \quad \psi(z) \equiv \mu+e \varphi_{1}(z)
$$

However, in this case equation (57) cannot be solved analytically, and the numerical integration methods are needed.

Let us show in the present section of the paper the effect of the plane dielectric surface charges on the obtained results. It is well known that infinitely thin homogeneously charged plate with charge density $\sigma(\xi)$ induces the homogeneous field intensity $E_{\sigma}=2 \pi \sigma(\xi)$ in vacuum (in this case 
the expression $\sigma(\xi)$ shows that the surface plane is described by the equation $z=\xi)$. This field has the opposite direction in the opposite sides of the plane. In the case of a charged plane dielectric surface the situation is absolutely similar. E.g., the negatively charged dielectric surface induces the field intensity $E_{2 \sigma}=2 \pi|\sigma(\xi)| / \varepsilon$ in the dielectric and $E_{1 \sigma}=-2 \pi|\sigma(\xi)|$ above the dielectric surface (see equation (24)). As mentioned earlier in the present paper, we consider only the cases of the same signs of charges both on the dielectric surface and in the volume above it (in our case we consider the negative charges). In this case, the field induced by the surface charges repulses the volume charges from the surface. So, the results obtained in the present Section remain useful if we substitute the external electric field in vacuum $E$ for $E-2 \pi|\sigma(\xi)|$ in the expressions (31)-(56),

$$
E \rightarrow E-2 \pi|\sigma(\xi)|
$$

It is easy to see that it is necessary to satisfy the condition

$$
E_{0}-2 \pi|\sigma(\xi)|>0
$$

that provides the possibility of the equilibrium volume charge distribution existing above the dielectric surface in the repulsive field of the surface charges.

\section{The charge system above the spatially inhomogeneous dielectric surface}

As already mentioned, the spatial inhomogeneities can be caused by the surface heterogeneities or by the inhomogeneous charge distribution on it (or both reasons simultaneously, see equations (8), (11), (20), (24))). Let us consider the mentioned surface inhomogeneities that slightly distort the electric field induced by the charge system above the plane dielectric:

$$
\begin{array}{cl}
\varphi_{1}(z, \boldsymbol{\rho})=\varphi_{1}(z)+\tilde{\varphi}_{1}(z, \boldsymbol{\rho}), & \varphi_{2}(z, \boldsymbol{\rho})=\varphi_{2}(z)+\tilde{\varphi}_{2}(z, \boldsymbol{\rho}), \\
\left|\varphi_{1}(z)\right| \gg\left|\tilde{\varphi}_{1}(z, \boldsymbol{\rho})\right|, & \left|\varphi_{2}(z)\right| \gg\left|\tilde{\varphi}_{2}(z, \boldsymbol{\rho})\right|
\end{array}
$$

where $\varphi_{1}(z), \varphi_{2}(z)$ are the potentials above the dielectric and inside it, respectively, in the case of the ideally plane dielectric surface with the equation of the profile $z=\xi$ (see equations (16)-(18)). The obtained procedure for the potentials $\varphi_{1}(z), \varphi_{2}(z)$ and charge density $n(z)$ is described in detail in the previous section of the present paper, see equations (26)-(59).

The next problem concerns obtaining the potentials $\tilde{\varphi}_{1}(z, \boldsymbol{\rho})$ and $\tilde{\varphi}_{2}(z, \boldsymbol{\rho})$. For these potentials one can use the equation (25) and the boundary conditions (20), (24). In terms of the Fouriertransforms $\tilde{\varphi}_{1}(z, \mathbf{q}), \tilde{\varphi}_{2}(z, \mathbf{q})$ over coordinate $\boldsymbol{\rho}$ of the potentials $\tilde{\varphi}_{1}(z, \boldsymbol{\rho})$ and $\tilde{\varphi}_{2}(z, \boldsymbol{\rho})$

$$
\tilde{\varphi}_{1}(z, \boldsymbol{\rho})=\int \mathrm{d}^{2} q \exp (i \mathbf{q} \boldsymbol{\rho}) \tilde{\varphi}_{1}(z, \mathbf{q}), \quad \tilde{\varphi}_{2}(z, \boldsymbol{\rho})=\int \mathrm{d}^{2} q \exp (i \mathbf{q} \boldsymbol{\rho}) \tilde{\varphi}_{2}(z, \mathbf{q})
$$

the equations (25) have the following form:

$$
\begin{aligned}
& \frac{\partial^{2} \tilde{\varphi}_{1}(z, \mathbf{q})}{\partial z^{2}}-q^{2} \tilde{\varphi}_{1}(z, \mathbf{q})=4 \pi e^{2} \frac{\partial n(z)}{\partial \mu} \tilde{\varphi}_{1}(z, \mathbf{q}) \\
& \frac{\partial^{2} \tilde{\varphi}_{2}(z, \mathbf{q})}{\partial z^{2}}-q^{2} \tilde{\varphi}_{2}(z, \mathbf{q})=0 .
\end{aligned}
$$

According to equations (20), (24), the boundary conditions concerning these equations can be written as:

$$
\begin{aligned}
& \left\{\tilde{\varphi}_{1}(z, \mathbf{q})-\tilde{\varphi}_{2}(z, \mathbf{q})\right\}_{z=\xi}=\left\{\frac{\partial \varphi_{2}(z)}{\partial z}-\frac{\partial \varphi_{1}(z)}{\partial z}\right\}_{z=\xi} \tilde{\xi}(\mathbf{q}) \\
& -4 \pi\left\{\mathrm{e} n(z)+\frac{\partial \sigma(\xi)}{\partial \xi}\right\}_{z=\xi} \tilde{\xi}(\mathbf{q})-4 \pi \tilde{\sigma}(\mathbf{q} ; \xi)=\left\{\frac{\partial \tilde{\varphi}_{1}(z, \mathbf{q})}{\partial z}-\varepsilon \frac{\partial \tilde{\varphi}_{2}(z, \mathbf{q})}{\partial z}\right\}_{z=\xi}
\end{aligned}
$$


where $\tilde{\xi}(\mathbf{q}), \tilde{\sigma}(\mathbf{q} ; \xi)$ are the Fourier-transforms of the quantities $\tilde{\xi}(\boldsymbol{\rho})$ and $\tilde{\sigma}(\boldsymbol{\rho} ; \xi)$, respectively (see equations (14), (18)):

$$
\tilde{\xi}(\boldsymbol{\rho})=\int \mathrm{d}^{2} q \exp (\operatorname{iq} \boldsymbol{\rho}) \tilde{\xi}(\mathbf{q}), \quad \tilde{\sigma}(\boldsymbol{\rho} ; \xi)=\int \mathrm{d}^{2} q \exp (\operatorname{iq} \boldsymbol{\rho}) \tilde{\sigma}(\mathbf{q} ; \xi) .
$$

Let us consider the intensity perturbations of the electric fields caused by inhomogeneities of the dielectric surface rapidly decreasing at $z \rightarrow \pm \infty$. It is easy to see that the first equation in equation (61) in general case cannot be solved analytically. However, in two particular cases the analytical solution exists. In the first case, we solve equation (61) at $z \sim \xi$ setting $\partial n(z) / \partial \mu$ equal to its value on the plane surface, $z=\xi$ :

$$
\frac{\partial n(z)}{\partial \mu} \approx \frac{\partial n(\xi)}{\partial \mu}
$$

Such a consideration is possible in the case when the typical size of spatial inhomogeneities of the unperturbed charge density $n(z)$ is considerably larger than the typical size of the spatial inhomogeneities of the potential $\tilde{\varphi}_{1}(z, \mathbf{q})$ along $z$-axis:

$$
\left|\left(\frac{\partial n(z)}{\partial \mu}\right)^{-1} \frac{\partial}{\partial z} \frac{\partial n(z)}{\partial \mu}\right|_{z=\xi} \ll\left|\left\{\tilde{\varphi}_{1}(z, \mathbf{q})\right\}^{-1} \frac{\partial \tilde{\varphi}_{1}(z, \mathbf{q})}{\partial z}\right|_{z=\xi} .
$$

Let us return to the discussion of the condition (64) below.

Then, taking into account the assumption of rapidly fading field densities at $z \rightarrow \pm \infty$, the solution of equation (60) can be given in the following form:

$$
\tilde{\varphi}_{1}(z, \mathbf{q})=A_{1}(\mathbf{q}) \exp (-z b(q)), \quad \tilde{\varphi}_{2}(z, \mathbf{q})=A_{2}(\mathbf{q}) \exp (z q)
$$

where (see equation (64))

$$
b(q) \equiv \sqrt{q^{2}+4 \pi e^{2} \frac{\partial n(\xi)}{\partial \mu}},
$$

and $A_{1}(\mathbf{q}), A_{2}(\mathbf{q})$ are obtained from the boundary conditions (62). To this end, we put the expressions (66) into the boundary conditions (62) and obtain the following relations for the potentials $\tilde{\varphi}_{1}(z, \mathbf{q}), \tilde{\varphi}_{2}(z, \mathbf{q})$ :

$$
\begin{aligned}
\tilde{\varphi}_{1}(z, \mathbf{q})= & \frac{\exp (-(z-\xi) b(q))}{\varepsilon q+b(q)}\left\{\left[\varepsilon q\left(E_{1}(\xi)-E_{2}(\xi)\right)\right.\right. \\
& +4 \pi(\mathrm{e} n(\xi)+(\partial \sigma(\xi) / \partial \xi))] \tilde{\xi}(\mathbf{q} ; \xi)+4 \pi \tilde{\sigma}(\mathbf{q} ; \xi)\}, \\
\tilde{\varphi}_{2}(z, \mathbf{q})=- & \frac{\exp ((z-\xi) q)}{\varepsilon q+b(q)}\left\{\left[b(q)\left(E_{1}(\xi)-E_{2}(\xi)\right)\right.\right. \\
& -4 \pi(\operatorname{en}(\xi)+(\partial \sigma(\xi) / \partial \xi))] \tilde{\xi}(\mathbf{q} ; \xi)-4 \pi \tilde{\sigma}(\mathbf{q} ; \xi)\},
\end{aligned}
$$

where (see equations (22), (26))

$$
E_{1}(\xi) \equiv-\left(\frac{\partial \varphi_{1}(z)}{\partial z}\right)_{z=\xi}, \quad E_{2}(\xi) \equiv-\left(\frac{\partial \varphi_{2}(z)}{\partial z}\right)_{z=\xi} .
$$

Let us emphasize that according to the boundary conditions (20), (21) (see also equations (58), (59)) the values of the quantities $E_{1}(\xi), E_{2}(\xi)$ can be expressed as

$$
E_{1}(\xi)=E_{0}-2 \pi|\sigma(\xi)|>0, \quad E_{2}(\xi)=\left(E_{0}+2 \pi|\sigma(\xi)|\right) / \varepsilon,
$$

where the field intensity $E_{0}$ is defined by the relation (36):

$$
E_{0}=E+4 \pi \mathrm{e} n_{s} .
$$


Let us recall that the values of the potentials $\tilde{\varphi}_{1}(z, \mathbf{q}), \tilde{\varphi}_{2}(z, \mathbf{q})$ at $z=\xi$ do not coincide due to the fact that the potential continuity on the surface in the case of its inhomogeneous wavy structure is provided by the inequalities (see equations (16)-(20)):

$$
\left.\varphi_{1}(z)\right|_{z=\xi}=\left.\varphi_{2}(z)\right|_{z=\xi}, \quad \delta \varphi_{1}(\xi, \mathbf{q})=\delta \varphi_{2}(\xi, \mathbf{q})
$$

where $\delta \varphi_{1}(\xi, \mathbf{q}) \equiv \tilde{\xi}(\mathbf{q})\left(\partial \varphi_{1}(z) / \partial z\right)_{z=\xi}+\tilde{\varphi}_{1}(\xi, \mathbf{q})$. According to equation (68), one can get:

$$
\begin{aligned}
\delta \varphi_{1}(\xi, \mathbf{q})=- & \frac{1}{\varepsilon q+b(q)}\left\{\left[\varepsilon q E_{2}(\xi)+b(q) E_{1}(\xi)-\right.\right. \\
& 4 \pi(\mathrm{e} n(\xi)+(\partial \sigma(\xi) / \partial \xi))] \tilde{\xi}(\mathbf{q} ; \xi)-4 \pi \tilde{\sigma}(\mathbf{q} ; \xi)\}
\end{aligned}
$$

where $E_{1}(\xi), E_{2}(\xi)$ are still defined by the relations (69). It is easy to see from the obtained formulae (68), (70) that the gas of the volume charges can sufficiently effect the potential of the electric field near the dielectric surface.

Now let us show that the solution of equation (61) in the forms (66), (68) is correct. As it is mentioned above, the condition of the existence of such solution is defined by the relation (65). According to equation (67) it can be expressed as follows:

$$
\left|\left(\frac{\partial n(\xi)}{\partial \mu}\right)^{-1} \frac{\partial}{\partial z} \frac{\partial n(\xi)}{\partial \mu}\right| \ll \sqrt{q^{2}+4 \pi e^{2} \frac{\partial n(\xi)}{\partial \mu}} .
$$

An explicit expression for the derivative $\partial n(\xi) / \partial \mu$ can be obtained from equations (31), (35), (36), (41), (46), (52), (56) both in the case of degenerate charge gas above the dielectric surface and in the case of nondegenerate one. In the second case the condition (71) has a rather simple form:

$$
q^{2} \gg \beta^{2} \mathrm{e}^{2}\left\{\left(E+2 \pi \mathrm{e} n_{s}\right)^{2}+4 \pi^{2} \mathrm{e}^{2} n_{s}^{2}\right\} .
$$

In the case, when the gas of charged Fermi-particles is degenerate at $z \ll z_{1}$ (see equation (55)) and low temperature expansions (52) take place, we can obtain the following expressions for the volume charge density $n(z)$ at $z \sim \xi$ and the electrochemical potential $\psi_{0}$ at $z=\xi$ (see equations (35), $(52),(56))$ :

$$
n(z) \approx \frac{2}{3} \nu \psi^{3 / 2}, \quad \psi_{0} \approx\left\{\frac{15}{32} \frac{E_{0}^{2}-E^{2}}{\pi \nu}\right\}^{2 / 5}
$$

where $\nu$ and $\psi$ are still defined by the relations (28) with $Q=-\mathrm{e}$, and $E_{0}, E$ are expressed by (36). Using the expression (73), one can write the condition (71):

$$
E^{2}-52 \pi \mathrm{e} n_{s} E-8 \pi^{2} \mathrm{e}^{2} n_{s}^{2} \ll 4 q^{2} \mathrm{e}^{-2}\left\{\frac{15}{4} \frac{\mathrm{e} n_{s}\left(E+2 \pi \mathrm{e} n_{s}\right)}{\nu}\right\}^{4 / 5} .
$$

Taking into account the solutions $(68)$ and the Fourier-transforms of the potentials $\tilde{\varphi}_{1}(z, \boldsymbol{\rho})$, $\tilde{\varphi}_{2}(z, \boldsymbol{\rho})$ (see equation $(60)$ ), the relations $(71)-(74)$ in a general case are correct for any value of $q$, including the value $q=0$ as well. It is easy to see that the relation (72) does not satisfy such a requirement. The relation (74) can take place at all values of $q$ in the case of the external pressing field $E$ which satisfies the following inequality:

$$
0 \leqslant E \leqslant E^{\prime}, \quad E^{\prime} \approx 52 \pi \mathrm{e} n_{s} .
$$

At $E>E^{\prime}$ the expressions (68) do not take place. In this case, as in the case of the condition (72) realization, the equations (61) should be solved using the numerical methods.

The case of particular interest is the spatially periodic inhomogeneities caused by the dielectric surface. As it is mentioned in the present paper, such inhomogeneities concern the twodimensional Wigner crystallization. In the simplest case of spatial periodic inhomogeneities, the 
Fourier-transforms of the quantities $\tilde{\xi}(\boldsymbol{\rho}), \tilde{\sigma}(\boldsymbol{\rho} ; \xi)$ (see equation $(63)$ ) can be expressed in the form:

$$
\begin{aligned}
\tilde{\sigma}(\mathbf{q} ; \xi) & =\frac{1}{2} \sum_{\alpha=1}^{2} \tilde{\sigma}\left(\mathbf{q}_{\alpha \sigma} ; \xi\right)\left\{\delta\left(\mathbf{q}+\mathbf{q}_{\alpha \sigma}\right)+\delta\left(\mathbf{q}-\mathbf{q}_{\alpha \sigma}\right)\right\} \\
\tilde{\xi}(\mathbf{q}) & =\frac{1}{2} \sum_{\alpha=1}^{2} \tilde{\xi}\left(\mathbf{q}_{\alpha \xi}\right)\left\{\delta\left(\mathbf{q}+\mathbf{q}_{\alpha \xi}\right)+\delta\left(\mathbf{q}-\mathbf{q}_{\alpha \xi}\right)\right\}
\end{aligned}
$$

where $\mathbf{q}_{\alpha \sigma}(\alpha=1,2)$ are the vectors of the reciprocal two-dimensional lattice concerning the spatial periodic charge distribution on the dielectric surface, $\mathbf{q}_{\alpha \xi}(\alpha=1,2)$ are the vectors of the reciprocal two-dimensional lattice concerning the spatial periodic wavy surface type, and $\tilde{\sigma}\left(\mathbf{q}_{\alpha \sigma} ; \xi\right)$, $\tilde{\xi}\left(\mathbf{q}_{\alpha \xi}\right)$ are the amplitudes of the corresponding surface heterogeneities. Of course, it is necessary to consider that the conditions (14) take place, which in this case can be written as:

$$
q_{\alpha \xi} \tilde{\xi}\left(\mathbf{q}_{\alpha \xi}\right) \ll 1, \quad q_{\alpha \sigma} \tilde{\xi}\left(\mathbf{q}_{\alpha \sigma}\right) \ll 1 .
$$

Then, putting the expressions (76) into equation (68) and making inverse Fourier transformation according to equation (60), it is easy to obtain the following expressions for the potentials $\tilde{\varphi}_{1}(z, \boldsymbol{\rho})$, $\tilde{\varphi}_{2}(z, \boldsymbol{\rho})$ :

$$
\begin{aligned}
\tilde{\varphi}_{1}(z, \boldsymbol{\rho})= & 4 \pi \sum_{\alpha=1}^{2} \frac{\exp \left(-(z-\xi) b\left(q_{\alpha \sigma}\right)\right)}{\varepsilon q_{\alpha \sigma}+b\left(q_{\alpha \sigma}\right)} \tilde{\sigma}\left(\mathbf{q}_{\alpha \sigma} ; \xi\right) \cos \left(\mathbf{q}_{\alpha \sigma} \boldsymbol{\rho}\right) \\
& +\sum_{\alpha=1}^{2} \frac{\exp \left(-(z-\xi) b\left(q_{\alpha \xi}\right)\right)}{\varepsilon q_{\alpha \xi}+b\left(q_{\alpha \xi}\right)}\left\{\varepsilon q_{\alpha \xi}\left(E_{1}(\xi)-E_{2}(\xi)\right)\right. \\
& +4 \pi(\operatorname{en}(\xi)+(\partial \sigma(\xi) / \partial \xi))\} \tilde{\xi}\left(\mathbf{q}_{\alpha \xi}\right) \cos \left(\mathbf{q}_{\alpha \xi} \boldsymbol{\rho}\right), \\
\tilde{\varphi}_{2}(z, \boldsymbol{\rho})= & 4 \pi \sum_{\alpha=1}^{2} \frac{\exp \left(-(z-\xi) q_{\alpha \sigma}\right)}{\varepsilon q_{\alpha \sigma}+b\left(q_{\alpha \sigma}\right)} \tilde{\sigma}\left(\mathbf{q}_{\alpha \sigma} ; \xi\right) \cos \left(\mathbf{q}_{\alpha \sigma} \boldsymbol{\rho}\right) \\
& -\sum_{\alpha=1}^{2} \frac{\exp \left(-(z-\xi) q_{\alpha \xi}\right)}{\varepsilon q_{\alpha \xi}+b\left(q_{\alpha \xi}\right)}\left\{b\left(q_{\alpha \xi}\right)\left(E_{1}(\xi)-E_{2}(\xi)\right)\right. \\
& -4 \pi(\operatorname{en}(\xi)+(\partial \sigma(\xi) / \partial \xi))\} \tilde{\xi}\left(\mathbf{q}_{\alpha \xi}\right) \cos \left(\mathbf{q}_{\alpha \xi} \boldsymbol{\rho}\right) .
\end{aligned}
$$

The obtained expressions represent the solution of the potential distribution problem (so, the charge density distribution, too) in the area near the dielectric surface with the weak (see equation (77)) spatially periodic inhomogeneities. Let us emphasize that for the validity of the expressions (78) it is no longer necessary to satisfy the conditions (71) for all the values of $q$. Its sufficiency is provided by the accomplishment of the conditions (71) (or equations (72), (74)) for two-dimensional reciprocal lattice distances $q_{\alpha \sigma}, q_{\alpha \xi}$.

Let us recall that we consider the simplest type of the spatial periodic inhomogeneities related to the dielectric surface. In the case of a more complicated structure of spatial-periodic homogeneities it is necessary to use the coefficients of two-dimensional Fourier series expansion for $\tilde{\xi}(\boldsymbol{\rho})$ and $\tilde{\sigma}(\boldsymbol{\rho} ; \xi)$, which describe these inhomogeneities.

\section{Conclusion}

Thus, the problem of an equilibrium state of the charged particles above the dielectric surface is solved. Equilibrium distributions for the charge and electric field induced by these charges in the system are obtained both in the case of ideally plane dielectric surface and in the case of weak spatial inhomogeneities that concern the dielectric surface. The weak spatial inhomogeneities caused both by the inhomogeneities of the surface itself and by the inhomogeneous charge distributions on it are taken into account. The case of "wavy" surface, in particular, the spatially periodic one, 
concerns the possible presence of the surface charge on it being taken into account. The effect of the external pressing electric field acting on the system is also taken into consideration. It is shown that the presence of the gas of volume charges essentially effects the value of the electric field potential in the area near the dielectric surface. Mostly, this fact plays an important role in describing the deformation of the liquid dielectric surface caused by near-surface charges pressure on it. The authors of the present paper are working at this problem at the moment.

However, in our opinion, the problem being solved is useful not only regarding the twodimensional Wigner crystallization aspect. The problem is worth solving for purely academic purposes because it can be related to the number of classical problems of electrodynamics and statistical physics. Due to this fact, in this paper we do not use the results of the real experiments on two-dimensional Wigner crystallization research. The formulations and the results obtained in this paper can be used for the research of the effect of the volume charges near the liquid helium surface on the spatial inhomogeneous states of the charges, which are adsorbed on the helium surface at the system parameters close to the experimental ones.

\section{Acknowledgements}

Authors acknowledge financial support from the Consolidated Foundation of Fundamental Research of Ukraine under grant No. 25.2/102 and thank S.V. Peletminsky for valuable discussions.

\section{References}

1. Wigner E., Phys. Rev., 1934, 46, No. 11, 1002.

2. Peletminsky A.S., Peletminsky S.V., Slusarenko Yu.V., Low Temp. Phys., 1999, 25, No. 5, 303.

3. Monarkha Yu.P., Shikin V.B., Sov. J. Low Temp. Phys., 1982, 8, No. 6, 279.

4. Monarkha Y., Kono K. Two-dimensional Coulomb Liquids and Solids. Springer-Verlag, Berlin, 2003, 346 .

5. Cole M.W., Cohen M.H., Phys. Rev. Lett., 1969, 23, No. 21, 1238.

6. Shikin V.B., JETP, 1970, 58, No. 5, 1748 (in Russian).

7. Brown T.R., Grimes C.C. Phys. Rev. Lett., 1972, 29, No. 18, 1233.

8. Grimes C.C., Brown T.R., Phys. Rev. Lett., 1974, 32, No. 6, 280.

9. Edelman V.S., Sov. Phys. Usp., 1980, 23, No. 4, 227.

10. Tamm I.E. Fundamentals of the Theory of Electricity. Central Books Ltd, 1980, 684 p.

11. Landau L.D., Lifshitz E.M. Statistical Physics, 3rd Ed. Pergamon Pres, Oxford, 1981 [Nauka, Moscow, 1980]. 


\title{
Про рівноважний розподіл електричних зарядів над поверхнею діелектриків
}

\author{
Д.М.Литвиненко, Ю.В.Слюсаренко \\ Інститут теоретичної фізики ім. А.І. Ахієзера ННЦ ХФТІ, вул. Академічна, 1, Харків, Україна \\ Отримано 27 грудня 2008 р.
}

Сформульовано задачу про рівноважний стан системи багатьох заряджених частинок над поверхнею діелектрику як у присутності зовнішнього притягувального електричного поля, так і при його відсутності. Отримано рівноважні розподіли зарядів та електричного поля, що виникає завдяки цим зарядам, у випадку ідеально плоскої поверхні діелектрику. Також отримані розв'язки рівнянь електростатики для досліджуванної системи у випадку малих просторових неоднорідностей, пов'язаних із поверхнею діелектрику. Відмічені просторові неоднорідності можуть бути пов'язані як із неоднорідностями самої поверхні, так і з неоднорідним розподілом заряду на ній. Розглянуто також випадок хвилястої, зокрема, просторово-періодичної поверхні з урахуванням можливості існування на ній просторових зарядів.

Ключові слова: заряджені ферміони, поверхня, твердий та рідкий діелектрики, рівноважні розподіли зарядів та електричного поля

PACS: $05.30 . F k, 05.70 . N p, 41.20 . C v, 71.10 . C a, 73.20 . A t$ 